\title{
A Influência das Práticas Maternas no Desenvolvimento Motor de Crianças Prematuras
}

\section{The Influence of Maternal Practices on the Motor Development of Premature Children}

\section{RESUMO}

Objetivo: Avaliar a influência das práticas maternas no desenvolvimento neuropsicomotor de crianças prematuras cadastradas no Ambulatório de Alto Risco do Centro Clínico da Universidade de Caxias do Sul. Metodologia: Participaram do estudo 74 crianças prematuras de 0 a 12 meses de idade corrigida, as quais foram avaliadas por meio da Alberta Infant Motor Scale (AIMS). Para análise dos dados foi utilizada estatística descritiva e o teste do Qui-quadrado de Pearson $(p=0,05)$. Resultados: Os resultados do estudo mostraram que $77 \%$ das crianças eram cuidadas pela mãe, poucas frequentavam a creche e a maioria permanecia pouco tempo na postura prono. O escore e o percentil médio da amostra foi de 14,81 ( $D P=9,50)$ e 37,18 (DP=26,674), classificando $44,6 \%$ da amostra com desenvolvimento motor atípico para a idade e $55,4 \%$ com desempenho adequado. Observou-se significância estatística na associação entre a categorização de desempenho da criança com o grau de escolaridade materna $(\mathrm{Chi} 2=5,273 ; \mathrm{p}=0,02)$ e o principal cuidador (Chi2=4,442; $p=0,035)$. Conclusão: Constatou-se que os fatores ambientais geram importante impacto nas aquisições motoras de crianças prematuras, principalmente o nível de escolaridade materna e a frequência da criança na creche.

\section{DESCRITORES}

Desenvolvimento Infantil. Prematuridade. Fator de Risco. Habilidades Motoras.

\begin{abstract}
Objective: Evaluate the influence of maternal practice on the neuropsychomotor development of premature infants registered at the High-risk Outpatient Clinic of the Clinical Center of the Caxias do Sul University. Methodology: 74 premature children with corrected age of 0 to 12 months participated in the study, which were assessed using the Alberta Infant Motor Scale (AIMS). For data analysis, descriptive statistics and Pearson's chi-square test were used $(p=0.05)$. Results: The results of the study showed that $77 \%$ of the children were cared for by the mother, few attended the daycare center and most remained in the prone position for a short time. The sample's average score and percentile was $14.81(\mathrm{SD}=9.50)$ and $37.18(\mathrm{SD}=$ 26.674 ), classifying $44.6 \%$ of the sample with atypical motor development for age and $55.4 \%$ with adequate performance. There was statistical significance in the association between the categorization of the child's performance and the level of maternal education (Chi2 $=5,273 ; p=0,02$ ) and the main caregiver (Chi2 $=4,442 ; p=0,035)$. Conclusion: it was found that environmental factors have an important impact on motor acquisitions of premature infant, especially the level of maternal education and the frequency of the child in daycare.
\end{abstract}

\section{DESCRIPTORS}

Child Development. Prematurity. Risk Factor. Motor Skills.

${ }^{1}$ Acadêmica do Curso de Fisioterapia da Universidade de Caxias do Sul, Ciências da Vida, Caxias do Sul, RS-Brasil.

${ }_{2}^{2}$ Fisioterapeuta graduada na Universidade de Caxias do Sul Caxias do Sul, RS-Brasil; Mestranda em Ciências da Saúde, Ciências da Vida, Universidade de Caxias do Sul, Caxias do Sul, RS-Brasil.

${ }^{3}$ Pós-Doutora em Ciências do Movimento Humano - ESEFID/Universidade Federal do Rio Grande do Sul, Porto Alegre, RS-Brasil; Docente do Curso de Fisioterapia da Universidade de Caxias do Sul, Ciências da Vida, Caxias do Sul, RS-Brasil. 
O desenvolvimento neuropsicomotor é um processo contínuo de aquisições de habilidades motoras e cognitivas que ocorre, principalmente, na primeira infância ${ }^{1}$. Durante esse processo, fatores intrínsecos e extrínsecos podem afetar o desenvolvimento motor de forma positiva ou negativa, considerando que o organismo da criança se molda conforme o ambiente onde ela está inserida ${ }^{2}$. O nível de escolaridade dos pais, o nível socioeconômico, a qualidade dos estímulos e as práticas maternas caracterizam os fatores extrínsecos ou ambientais, já a prematuridade, o baixo peso ao nascer e complicações no nascimento são considerados fatores biológicos ou intrínsecos ${ }^{3}$.

Dentre os fatores de risco, sabe-se que o nível de conhecimento dos pais sobre o desenvolvimento motor está associado a melhores habilidades parentais e estímulos apropriados para os filhos, exercendo uma influência positiva no desenvolvimento, diminuindo assim o risco de comorbidades ${ }^{4}$. São exemplos de práticas maternas, a amamentação, o conhecimento dela sobre o desenvolvimento motor e as práticas educativas e de estimulação. Mães com menor nível educacional demonstram habilidades parentais menores; já o conhecimento dos marcos do desenvolvimento está relacionado com a utilização de novas experiências e brincadeiras com os filhos ${ }^{4,5}$ gerando diferentes estímulos para o seu desenvolvimento.

A prematuridade é um fator de risco importante no desenvolvimento neuropsicomotor, tendo em vista que gera uma suspensão no desenvolvimento das estruturas anatômicas da criança ${ }^{6}$. O nascimento prematuro está associado com atrasos no desenvolvimento, como distúrbios intelectuais, comportamentais e de linguagem, quando comparados aos lactentes nascidos a termo ${ }^{7}$. Tendo em vista que a prematuridade é um insulto ao desenvolvimento, as práticas parentais adequadas são consideradas fundamentais para a aquisição dos marcos motores do desenvolvimento na idade adequada, evitando que alterações motoras se instalem ${ }^{4}$.

Diante disso, é de suma importância estudos que avaliem o desenvolvimento motor de crianças expostas a diferentes fatores de risco, tendo em vista que os mesmos podem ser minimizados quanto mais cedo for sua identificação. Desta forma, medidas interventivas podem ser viabilizadas e implementadas, evitando atrasos no desenvolvimento motor infantil. Sendo assim, o objetivo do presente estudo foi avaliar a influência das práticas maternas no desenvolvimento motor de crianças prematuras, com idade corrigida de 0 a 12 meses, cadastradas em um Ambulatório de Alto Risco na cidade de Caxias do Sul.

\section{METODOLOGIA}

Trata-se de uma pesquisa descritiva e observacional, de caráter associativo, com abordagem transversal, aprovado pelo Comitê de Ética em Pesquisa da Universidade de Caxias do Sul (UCS) (n 2.688.022).

Participaram do estudo 74 bebês pré-termos cadastrados no Ambulatório de Alto Risco do Centro Clínico da Universidade de Caxias do Sul. Os critérios de inclusão estabelecidos foram: a) os pais ou responsáveis consentir e assinarem o Termo de Consentimento Livre e Esclarecido (TCLE); b) ser encaminhado da UTI Neonatal do 
Hospital Geral de Caxias do Sul; c) pré-termos (nascidos antes de 37 semanas de gestação) com idade corrigida até 12 meses; d) ser avaliado pelos profissionais da fisioterapia no Ambulatório de Alto Risco. Já os critérios de exclusão foram: a) incapacidade de realizar a avaliação; b) diagnóstico de doença neurológica confirmada; c) alterações de sinais vitais; d) sono profundo; e) choro intenso; f) não consentimento dos pais ou responsáveis. A partir desses critérios foram excluídos 39 participantes, sendo $18 \mathrm{com}$ diagnóstico de doença neurológica, 12 crianças nascidas a termo, 7 com idade acima de 14 meses e 2 por não completarem a avaliação.

Para a avaliação do desenvolvimento motor das crianças foi utilizada a Alberta Infant Motor Scale (AIMS), que foi elaborada por Piper e Darrah ${ }^{8}$ no Canadá, com bebês desde o nascimento até os 18 meses de idade. A escala possui 58 itens divididos nas posturas prono (21 itens), supino (9 itens), sentado (12 itens) e em pé (16 itens), as quais retratam a movimentação espontânea e a sequência de aquisições motoras do desenvolvimento infantil. É um instrumento observacional da motricidade ampla que, em cada item é observado onde ocorre a sustentação de peso e o movimento antigravitacional ou voluntário que o bebê realiza na posição ${ }^{9}$ desconsidera reflexos e permite apenas pouco manuseio do avaliador. A escala fornece um escore bruto de 0 a 58 pontos, obtido através da soma do escore de cada postura e é convertido em um percentil. Posteriormente os percentis são agrupados em categorias de desempenho motor, sendo abaixo de $5 \%$ considerado com desenvolvimento anormal, entre $5 \%$ e $25 \%$ com suspeita no desenvolvimento, e acima de $25 \%$ desempenho motor normal $\left.\right|^{9,10}$

A AIMS é utilizada para acompanhar os ganhos motores dos bebês, identificar atrasos ou anormalidades e, também, avaliar a eficiência das intervenções em crianças com disfunções ou atrasos no desenvolvimento ${ }^{9}$. É uma ferramenta valiosa que foi validada e normatizada para a população brasileira, que é amplamente utilizada por pesquisadores e profissionais da saúde, devido a sua praticidade e características psicométricas ${ }^{11}$.

Também foram utilizados dois questionários para as variáveis relacionadas a fatores de riscos biológicos e ambientais, tais como: sexo, idade cronológica e corrigida, idade gestacional, peso e comprimento ao nascer, perímetro cefálico, idade do pai e da mãe, número de filhos, renda familiar, escolaridade dos pais, se a criança fica em prono, alimentação atual e internações hospitalares.

As crianças, acompanhadas dos pais ou responsáveis, passaram pela equipe da fisioterapia (composta por três acadêmicas e duas fisioterapeutas) em uma sala do Ambulatório de Alto Risco. Inicialmente foi explicado para os pais o processo de avaliação e, mediante a assinatura do TCLE, eram realizadas as coletas de dados em prontuário e carteira nacional de vacinação, além da aplicação dos questionários da pesquisa. Depois, foi iniciada a avaliação com a AIMS, com duração média de 20 minutos. Na avaliação, a criança foi observada nas diferentes posturas da escala, com a menor quantidade de roupa possível e mínimo manuseio dos avaliadores, utilizando apenas estímulos sensório-motores através de brinquedos sonoros e coloridos. Os avaliadores foram previamente treinados com o instrumento de avaliação até alcançar a con- 
cordância interavaliadores indicado pela escala. Finalizada a avaliação, as pontuações eram definidas e realizadas orientações aos pais, de acordo com a idade motora da criança.

Os dados coletados foram analisados pelo programa estatístico SPSS versão 20.0. Foi utilizada estatística descritiva com distribuição de frequência simples e relativa, bem como as medidas de tendência central (média/ mediana) e de variabilidade (desvio padrão). Para as associações da categorização de desempenho e variáveis ambientais adotou-se o teste Qui-quadrado de Pearson, com nível de significância de 5\%. Para associação de percentil com as variáveis ambientais foi aplicado o teste $\mathrm{Eta}^{2}$, considerando associação fraca resultados abaixo de 0,30 , associação média entre 0,30 e 0,60 e associação forte para resultados acima de 0,60.

\section{RESULTADOS}

Em relação às características biológicas gerais dos 74 participantes ( 35 meninas e 39 meninos), observou-se que $81,1 \%(n=60)$ apresentava até 6 meses de idade corrigida e 18,9\% ( $n=14)$ entre 7 e 12 meses de idade corrigida e $18,9 \%(n=14)$ da amostra era gemelar. No que se refere aos fatores de risco biológico, destaca-se ainda que a maioria da amostra nasceu com muito baixo peso e prematuridade moderada. Quanto ao tipo de parto, $68,9 \%(n=51)$ nasceram de cesárea. $A$ descrição de todas as características biológicas está disposta na Tabela 1.

As características ambientais mostram que a maior frequência foi de pais com renda familiar de 1 a 2 salários e com nível de escolaridade baixo. Como características de práticas maternas, foi observado como principal cuidador a mãe $(77 \%)$ e poucos frequentavam a creche $(5,4 \%)$. Além disso, as crianças ficavam pouco na postura prono e o decúbito lateral foi o mais relatado como postura adotada para dormir (52,7\%). A análise mais detalhada das variáveis ambientais e maternas encontram-se na Tabela 2.

Quanto ao desempenho motor dos bebês, verificou-se que $14,9 \%$ foram classificados com desenvolvimento motor atrasado, $29,7 \%$ apresentaram desenvolvimento motor suspeito e $55,4 \%$ demonstraram desenvolvimento motor normal para a idade corrigida. No que se refere ao escore bruto e percentil, o escore médio da amostra foi de 14,81 (DP=9,50) e o percentil de 37,18 (DP=26,674).

A associação do percentil com as variáveis ambientais e de práticas maternas (tabela 3) se mostrou moderada, indicando que o desempenho motor sofre influência destas. Ao relacionar o percentil de desempenho com o fato da criança ir para a creche, observou-se uma associação moderada (Eta=0,74; $\left.\mathrm{Eta}^{2}=0,54\right)$, indicando que bebês que frequentavam a creche apresentaram meIhor desempenho motor e 39\% daquelas que não frequentam creche apresentaram desenvolvimento inadequado. A quantidade de filho que a mãe do bebê tem apresenta associação moderada com o percentil de desempenho motor ( $E t a=0,57 ; \mathrm{ta}^{2}=0,32$ ), indicando que quanto maior a quantidade de filhos, pior o desenvolvimento. Notou-se ainda, que as crianças que nunca eram deixadas em prono ou ficavam poucas vezes, demonstraram desenvolvimento inferior aos que ficavam nesta posição $\left(E t a=0,67 ; E^{2}{ }^{2}=0,44\right)$.

A relação entre a categorização do 
Tabela 1. Características biológicas dos bebês.

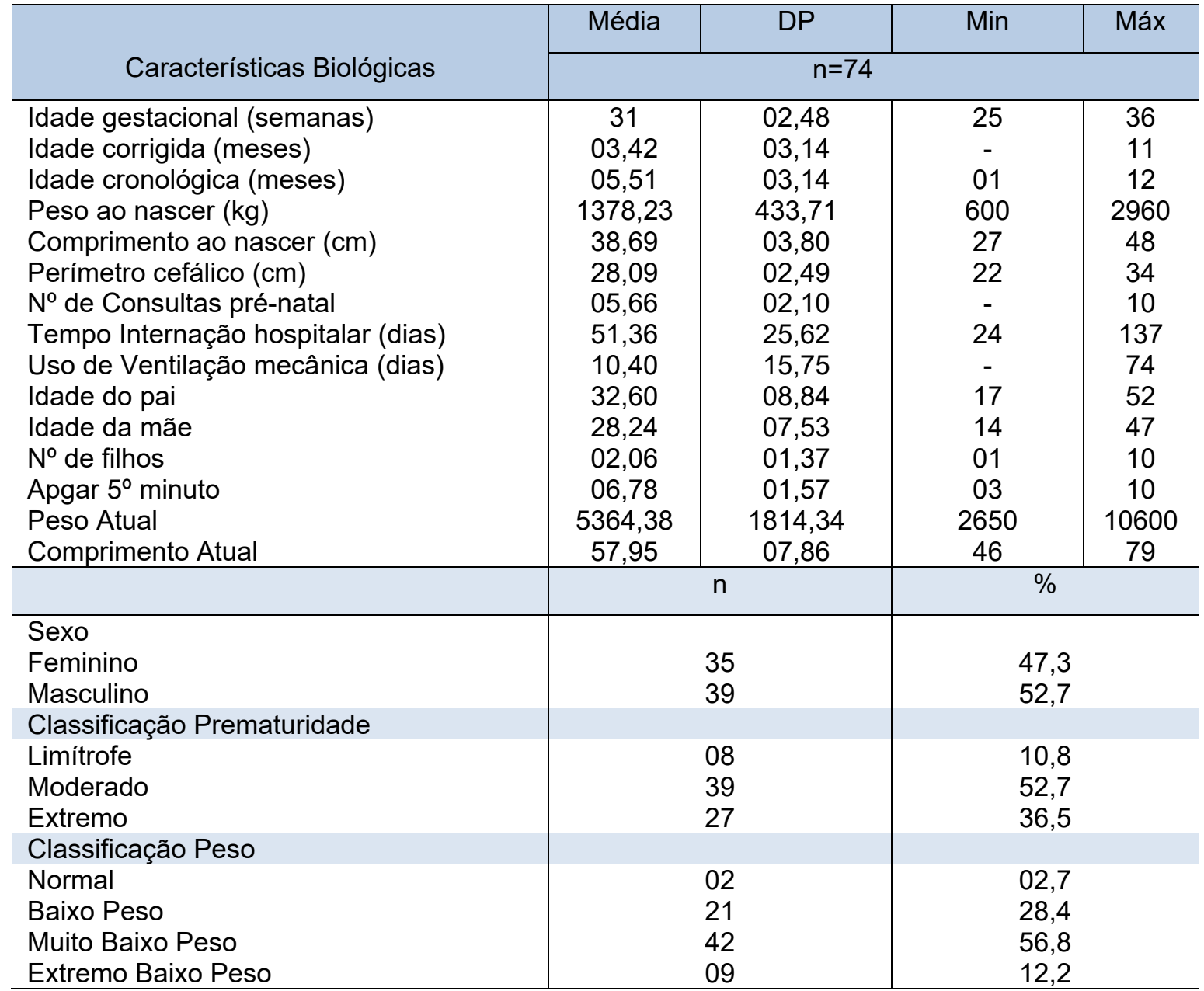

Legenda: $\mathrm{n}=$ número; $\mathrm{FR}=$ frequência; min=mínimo; máx=máximo; $\mathrm{kg}=$ =quilogramas; $\mathrm{cm}=$ centímetros.

desempenho motor e o grau de escolaridade da mãe indicou associação significativa $\left(\mathrm{Chi}^{2}=5,273 ; \mathrm{p}=0,02\right)$, o que indica que quanto menor o grau de escolaridade da mãe, pior é o desempenho da criança. Relacionando com o principal cuidador, a mesma associação significativa foi identificada $\left(\mathrm{Chi}^{2}=4,442 ; \mathrm{p}=0,035\right)$, expondo que parte das crianças cuidadas pela mãe demonstraram desenvolvimento atípico. A relação da categorização e do percentil de desempenho com as variáveis ambientais e de práticas maternas estão expressas na tabela 3.

\section{DISCUSSÃO}

Este estudo procurou avaliar a influência das práticas maternas no desenvolvimento neuropsicomotor de crianças prematuras, verificando possíveis associações entre as práticas maternas e o desempenho motor das crianças. Os resultados demonstraram 
Tabela 2. Características Ambientais e Maternas.

\begin{tabular}{|c|c|c|}
\hline Características Ambientais e Maternas & $\mathrm{n}$ & $\%$ \\
\hline \multicolumn{3}{|l|}{ Renda em salários } \\
\hline 1 a 2 & 36 & 48,6 \\
\hline 3 a 4 & 25 & 33,7 \\
\hline 5 a 7 & 01 & 01,4 \\
\hline Renda variável & 01 & 01,4 \\
\hline \multicolumn{3}{|l|}{ Escolaridade do pai } \\
\hline Nunca estudou & 01 & 01,4 \\
\hline $1^{\circ} \mathrm{grau}$ incompleto & 21 & 28,4 \\
\hline $1^{\circ} \mathrm{grau}$ completo & 11 & 14,9 \\
\hline Ensino médio incompleto & 11 & 14,9 \\
\hline Ensino médio completo & 19 & 25,7 \\
\hline Ensino superior incompleto & 03 & 04,1 \\
\hline Ensino superior completo & 02 & 02,7 \\
\hline \multicolumn{3}{|l|}{ Escolaridade da mãe } \\
\hline $1^{\circ}$ grau incompleto & 15 & 20,3 \\
\hline $1^{\circ} \mathrm{grau}$ completo & 15 & 20,3 \\
\hline Ensino médio incompleto & 14 & 18,9 \\
\hline Ensino médio completo & 18 & 24,3 \\
\hline Ensino superior incompleto & 06 & 08,1 \\
\hline \multicolumn{3}{|l|}{ Principal Cuidador } \\
\hline Pai & 01 & 01,4 \\
\hline Mãe & 57 & 77 \\
\hline Pais & 07 & 9,5 \\
\hline Avó & 01 & 1,4 \\
\hline \multicolumn{3}{|l|}{ Frequenta Creche } \\
\hline Sim & 04 & 5,4 \\
\hline Não & 64 & 86,5 \\
\hline \multicolumn{3}{|l|}{ Fica em Prono } \\
\hline Nunca & 19 & 25,7 \\
\hline Às vezes & 31 & 41,9 \\
\hline Sempre & 09 & 12,2 \\
\hline Não utiliza & 01 & 01,4 \\
\hline \multicolumn{3}{|l|}{ Postura ao dormir } \\
\hline Prono & 02 & 02,7 \\
\hline Supino & 17 & 23 \\
\hline Lateral & 39 & 52,7 \\
\hline Variações & 04 & 05,4 \\
\hline
\end{tabular}

que diversos fatores de risco contribuíram para os atrasos no desenvolvimento motor observados na amostra.

Dentre os fatores de risco, a prematuridade é apontada como um dos fatores de risco mais associados ao atraso motor, principalmente nos primeiros 12 meses de vida, pelo fato dessas crianças terem os órgãos e sistemas imaturos ao nascimento ${ }^{12}$. Os prematuros estão expostos a fatores que geram influências decisivas no seu desenvolvimento, como sua vulnerabilidade biológica 
Tabela 3. Associação entre percentil/categorização e práticas maternas.

\begin{tabular}{lcc}
\hline Percentil & Eta & Eta $^{2}$ \\
\hline Grau de escolaridade & 0,56 & 0,31 \\
Frequenta Creche & 0,74 & 0,54 \\
Principal Cuidador & 0,62 & 0,39 \\
No de Filhos & 0,57 & 0,32 \\
Fica em prono & 0,67 & 0,44 \\
Posição ao dormir & 0,67 & 0,44 \\
\hline Categorização & $\mathrm{Chi}^{2}$ & $\mathrm{p}(0,05)$ \\
\hline Grau de escolaridade & 5,27 & $0,02^{*}$ \\
Frequenta Creche & 1,54 & 0,21 \\
Principal Cuidador & 4,44 & $0,035^{*}$ \\
No de Filhos & 0,11 & 0,73 \\
Fica em prono & 0,01 & 0,91 \\
Posição ao dormir & 1,29 & 0,25 \\
\hline
\end{tabular}

Legenda: $\mathrm{N}^{\circ}=$ número; ${ }^{*}=$ estatisticamente significativo.

e ao ambiente que estão inseridos ${ }^{13}$. Ainda, bebês prematuros com muito baixo peso ao nascer, sofrem ameaças de distúrbios motores e exprimem diferenças no desenvolvimento motor quando comparadas ao nascidos a termo ${ }^{14}$. Nesta pesquisa, a prematuridade e o baixo peso foram fatores que também podem ter interferido para os atrasos observados.

Um estudo prévio de Fuentefria, Silveira e Procianoy ${ }^{15}$ diz que os prematuros podem apresentar déficits de crescimento e desenvolvimento a longo prazo, particularmente os nascidos com menos de 32 semanas de gestação e de muito baixo peso, como grande parte da população avaliada neste estudo. Corroborando com o estudo anterior, Bélanger e colaboradores ${ }^{16}$ citam que pode haver comprometimento das habilidades motoras na infância de crianças prematuras, bem como interferir na capacidade de exploração, no envolvimento de atividades e na criação de novas habilidades motoras, sejam elas grossas ou finas. A conquista de habilidades motoras gera oportunidades para a interação com as pessoas e o ambiente, aprimorando comportamentos perceptivos, cognitivos, emocionais e sociais ${ }^{14}$. Além disso, essas crianças apresentam taxas elevadas de comprometimento motor e cognitivo que podem estar presentes na adolescência e na vida adulta, quando contrapostas a crianças a termo ${ }^{17}$.

A prematuridade e o baixo peso quando associados a outros fatores de risco, potencializam os atrasos motores na criança, aspecto este, observado no presente estudo, onde a amostra foi considerada de alto risco devido a somatória de diferentes fatores de risco. Sob o mesmo ponto de vista, um estudo de Ribeiro e colaboradore ${ }^{18}$ diz que as intercorrências pré, peri e pós-natais somadas a fatores socioeconômicos podem interferir no curso normal do desenvolvimento. Além disso, bebês prematuros expostos a vários fatores de risco apresentam maior vulnerabilidade contrapostos a crianças nascidas a termo ${ }^{19}$. Em vista disso, a avaliação do desempenho motor dessa população se faz importante, sabendo que essas habilidades demonstram 
a integridade e a maturação dos sistemas, além de servir como método de identificação e intervenção precoce para evitar possíveis complicações futuras ${ }^{20}$.

Foi constatado que as crianças com mães de menor grau de escolaridade apresentam piores desempenhos motores. Corroborando com os achados desta pesquisa, um estudo de Patra e colaboradores ${ }^{21}$ relata que o nível de escolaridade da mãe tem grande influência no desenvolvimento, além de ser um preditor de maior impacto do que outros fatores socioeconômicos, tendo em vista que mães com maior conhecimento sobre o desenvolvimento infantil realizam estímulos de maior qualidade ${ }^{5}$. Inesperadamente, notou-se desenvolvimento inadequado em $83,3 \%$ das crianças de mães com ensino superior incompleto, contradizendo estudos que relacionam o ensino superior com melhores cuidados infantis e desenvolvimento ${ }^{21,22}$. Isso porque, o nível de educação materna vem sendo considerado um fator de atenção ao desenvolvimento, tendo em vista que elevados níveis de escolaridade acarretam em jornadas de trabalhos mais longas, diminuindo assim o contato da mãe com o bebê e, consequentemente, o tempo de estímulo ${ }^{23}$.

No contexto atual, as famílias tem deixado seus filhos sob os cuidados de outros, devido a rotina de trabalho e por isso, as escolas de educação infantil se tornaram um fator ambiental que pode exercer influência sobre o desenvolvimento infantil, uma vez que a criança permanece grande parte do tempo nela ${ }^{24}$. No presente estudo observou-se que crianças que não frequentavam creche demonstraram pior desempenho. Indo de encontro com o estudo de Corsi et $\mathrm{al}^{3}$, no qual evidenciam que a creche oferece estímulos adicionais à criança, dado que ela vai interagir com outras crianças e cuidadores, porém isso está relacionado com a qualidade do estímulo ofertado, já as crianças que não frequentam a creche são privadas deste estímulo extra, vivenciando apenas os estímulos em seu domicílio.

Além da criança frequentar a creche, quem cuida dela também apresenta influência no seu desenvolvimento. Neste estudo verificou-se uma associação moderada entre o percentil de desempenho e o principal cuidador da criança, indicando que parte das crianças cuidadas pelas mães apresentaram desenvolvimento inadequado. Este achado pode ser explicado pelo fato das mães brasileiras serem super-protetoras, oferecerem muito colo e poucos momentos de estímulo em prono e sedestação ${ }^{22}$. Além disso, os cuidadores evitam colocar seus filhos na postura prona relatando desconforto para a criança, medo de sufocamento e por não saberem como ajudar a criança a suportar mais tempo na postura ${ }^{25}$.

Um estudo relata que privar o bebê da experiência em prono pode levar à atrasos nas aquisições de habilidades motoras futuras, além de gerar assimetrias no crânio ${ }^{25}$. E como verificado na presente pesquisa, os pais não tem costume de deixar seus filhos de barriga para baixo. Estudos demonstram que a postura prono é a mais importante no repertório motor da criança, visto que contribui para o desenvolvimento locomotor, cognitivo, habilidades espaciais e manipulativas, além de preparar a musculatura antigravitacional para a sedestação, a ortostase, dissociação de cinturas, coordenação, e ainda, fortalece a 
musculatura dos membros superiores e auxilia na capacidade de rolar $^{26,27}$.

Uma possível explicação para os pais não deixarem seus filhos em prono, é a campanha "Back to sleep" ou "Este lado para cima". Essa campanha foi criada a fim de reduzir a ocorrência da síndrome da morte súbita no bebê, incentivando os pais a colocarem seus filhos para dormir em supino, no entanto a campanha acabou afetando a hora de brincar em prono ${ }^{28}$. Um estudo realizado com 75 crianças avaliadas pela AIMS que investigou se o desenvolvimento motor grosso das crianças aos 6 meses é afetado pela posição preferida de dormir, concluiu que a posição de dormir não tem efeito significativo nas realizações motoras aos 6 meses $^{28,29}$. Carmeli e seus colaboradores ${ }^{29}$ relatam que mesmo bebês que dormem em supino mas usufruem da postura prono diariamente para brincar, apresentam as mesmas características motoras grossas que os bebês que dormem em prono. No presente estudo foi observado que a maioria das crianças dormiam em decúbito dorsal e lateral, não permanecendo em prono para brincar.

Foi verificado na presente pesquisa uma associação moderada entre a quantidade de filhos e desempenho motor, indicando que a maioria das crianças de famílias grandes

\section{REFERÊNCIAS}

1. Zago JTdC, Pinto PAF, Leite HR, Santos JN, Morais RLdS. Associação entre o desenvolvimento neuropsicomotor e fatores de risco biológico e ambientais em crianças na primeira infância. Revista CEFAC. 2017;19(3):320-329. apresentaram pior desempenho. Outros estudos demonstraram que famílias grandes são consideradas um fator de risco ao desenvolvimento neuropsicomotor, visto que não são ambientes estimulantes para o bebê ${ }^{30}$. Lamy Filho e colaboradores ${ }^{30}$ ainda afirmam que, em casas com muitos residentes, os brinquedos e a atenção dos pais ficam fragmentadas entre os filhos, diminuindo assim a possibilidade de estimulação adequada.

\section{CONCLUSÃO}

No presente estudo foi observado que os fatores ambientais geram impacto nas aquisições motoras, destacando-se o nível de escolaridade materna e a frequência na creche. Além disso, ao contrário do que deveria ser, a postura prona é a menos estimulada pelos pais e cuidadores. Dessa forma é preciso identificar crianças com fatores de risco para atraso, a fim de capacitar profissionais da área da saúde a identificá-los e realizar intervenções e orientações juntamente aos pais. Sugere-se ainda a realização de estudos futuros com acompanhamento longitudinal, visando verificar como esses fatores se comportam a longo prazo, podendo inferir com mais segurança, como ocorre o processo de desenvolvimento motor diante de diferentes fatores de influência. 
3. Corsi C, Santos MMd, Marques LdAP, Rocha NACF. Impact of extrinsic factors on fine motor performance of children attending day care. Rev Paulista de Pediatria. 2016;34(4):439-446.

4. Scarzello D, Arace A, Prino LE. Parental practices of Italian mothers and fathers during early infancy: The role of knowledge about parenting and child development. Infant Behav Dev. 2016; 44:133-143.

5. Bornstein MH, Cote LR, Haynes OM, Hahn CS, Park Y. Parenting knowledge: experiential and sociodemographic factors in European American mothers of young children. Dev Psychol. 2010; 46(6):1677-1693.

6. Araújo ATdC, Eickmann $\mathrm{SH}$, Coutinho SB. Fatores associados ao atraso do desenvolvimento motor de crianças prematuras internadas em unidade de neonatologia. Revista Brasileira de Saúde Materno Infantil. 2013;13(2):119-128.

7. Mansson J, Fellman V, Stjernqvist K. Extremely preterm birth affects boys more and socio-economic and neonatal variables pose sex-specific risks. Acta Paediatr. 2015;104(5):514-521

8. Piper MC, Pinnell LE, Darrah J, Maguire T, Byrne PJ. Construction and validation of the Alberta Infant Motor Scale (AIMS). Can J Public Health. 1992;83 Suppl 2:S46-50.

9. Valentini NC, Saccani R. Escala Motora Infantil de Alberta: validação para uma população gaúcha. Rev Paulista de Pediatria. 2011;29(2):231-238.

10. Valentini NC, Saccani R. Brazilian validation of the Alberta Infant Motor Scale. Phys Ther. 2012;92(3):440447.

11. Saccani R, Valentini NC, Pereira KR. New Brazilian developmental curves and reference values for the Alberta infant motor scale. Infant Behav Dev. 2016;45(Pt A):3846.

12. Saccani R, Valentini NC, Pereira KR, Muller AB, Gabbard C. Associations of biological factors and affordances in the home with infant motor development. Pediatr Int. 2013;55(2):197-203.

13. Moreira RS, Magalhaes LC, Alves CR. Effect of preterm birth on motor development, behavior, and school performance of school-age children: a systematic review. J Pediatr (Rio J). 2014;90(2):119-134.

14. Su YH, Jeng SF, Hsieh WS, Tu YK, Wu YT, Chen LC. Gross Motor Trajectories During the First Year of Life for Preterm Infants With Very Low Birth Weight. Phys Ther. 2017;97(3):365-373.

15. Fuentefria RDN, Silveira RC, Procianoy RS. Motor development of preterm infants assessed by the Alberta Infant Motor Scale: systematic review article. J Pediatr (Rio J). 2017;93(4):328-342.

16. Belanger R, Mayer-Crittenden C, Minor-Corriveau M, Robillard M. Gross Motor Outcomes of Children Born Prematurely in Northern Ontario and Followed by a Neonatal Follow-Up Programme. Physiother Can. 2018;70(3):233-239.

17. Olsen JE, Allinson LG, Doyle LW, Brown NC, Lee KJ,
Eeles AL et al. Preterm and term-equivalent age general movements and 1-year neurodevelopmental outcomes for infants born before 30 weeks' gestation. Dev Med Child Neurol. 2018;60(1):47-53.

18. Ribeiro CD, Pachelli MR, Amaral NC, Lamonica DA. Development skills of children born premature with low and very low birth weight. Codas. 2017;29(1):e20160058.

19. Fuentefria RN, Silveira RC, Procianoy RS. Neurodevelopment and Growth of a Cohort of Very Low Birth Weight Preterm Infants Compared to Full-Term Infants in Brazil. Am J Perinatol. 2018;35(2):152-162.

20. Formiga CKMR, Linhares MB. Motor development curve from 0 to 12 months in infants born preterm. Acta Paediatr. 2011;100(3):379-384.

21. Patra K, Greene MM, Patel AL, Meier P. Maternal Education Level Predicts Cognitive, Language, and Motor Outcome in Preterm Infants in the Second Year of Life. Am J Perinatol. 2016;33(8):738-744.

22. Pereira KRG, Saccani R, Valentini NC. Cognição e ambiente são preditores do desenvolvimento motor de bebês ao longo do tempo. Fisioterapia e Pesquisa. 2016;23(1):59-67.

23. Pereira KR, Valentini NC, Saccani R. Brazilian infant motor and cognitive development: Longitudinal influence of risk factors. Pediatr Int. 2016;58(12):1297-1306.

24. Silva ÂCDd, Engstron EM, Miranda CTd. Fatores associados ao desenvolvimento neuropsicomotor em crianças de 6-18 meses de vida inseridas em creches públicas do Município de João Pessoa, Paraíba, Brasil. Cad de Saúde Pública. 2015;31(9):1881-1893.

25. Palmer CF, Rindler D, Leverone B. Moving into tummy time, together: Touch and transitions aid parent confidence and infant development. Infant Ment Health $\mathrm{J}$. 2019;40(2):277-288.

26. Gerzson LR, Catarino BM, Azevedo KAd, Demarco PR, Palma MS, Almeida CSd. Frequência semanal de um programa de intervenção motora para bebês de berçário. Fisioterapia e Pesquisa. 2016;23(2):178-184.

27. Silveira RC, Mendes EW, Fuentefria RN, Valentini NC, Procianoy RS. Early intervention program for very low birth weight preterm infants and their parents: a study protocol. BMC Pediatr. 2018;18(1):268.

28. Wittmeier K, Mulder K. Time to revisit tummy time: A commentary on plagiocephaly and development. Paediatr Child Health. 2017;22(3):159-161.

29. Carmeli E, Marmur R, Cohen A, Tirosh E. Preferred sleep position and gross motor achievement in early infancy. Eur J Pediatr. 2009;168(6):711-715.

30. Lamy Filho F, Medeiros SMd, Lamy ZC, Moreira MEL. Ambiente domiciliar e alterações do desenvolvimento em crianças de comunidade da periferia de São Luís MA. Ciência \& Saúde Coletiva. 2011;16(10):4181-4187.

\section{CORRESPONDÊNCIA}

Raquel Saccani

Endereço: Rua Francisco Getúlio Vargas, 1130

Bairro Petrópolis - Caxias do Sul - RS, Brasil.

E-mail: rsaccani@ucs.br 